\title{
Polyamines in biological systems
}

\author{
Enzo Agostinelli
}

Published online: 15 December 2009

(C) Springer-Verlag 2009

In the present issue, the authors attempt to give the essence of their lectures and posters, including subjects of discussion, that were presented at the International Congress on Amino Acids, Peptides and Proteins held in Wien, August 3-7, 2009. The meeting was organized by professor G. Lubec and professor E. Agostinelli (as concerns the "Polyamines Session").

In this issue several aspects concerning the physiological roles of polyamines are also described by others internationally recognized experts that did not attend to the meeting. The articles deal with research carried out up to mid-2009 and contain literature references, figures, tables and reaction schemes. In fact, the issue is intended to provide a relatively short overview of some important concepts and notions on the polyamines and represents an important tool for those who set about to investigate on this intriguing field.

Polyamines are polycationic biogenic amines required for both eukaryotic and prokaryotic cell growth and differentiation and in the synthesis of proteins and nucleic acids. They attract interest because of their multiple functions in cell biology including, among many others, cell cycle regulation, gene expression and signal transduction.

During the last two decades, polyamines have been isolated from practically every microbial, plant, or animal cell analysed. Subsequently, considerable progress has been made in understanding their metabolic pathways and numerous enzymes involved in polyamines synthesis and degradation have been isolated and studied. In this period

\section{E. Agostinelli $(\bowtie)$}

Department of Biochemical Sciences “A. Rossi Fanelli”,

SAPIENZA University of Rome, Piazzale Aldo Moro 5,

00185 Rome, Italy

e-mail: enzo.agostinelli@uniroma1.it the literature, characterized by thousands of publications, has attested the interest of the scientific community towards these polycations.

The natural polyamines (putrescine, spermidine, spermine, and related structures) are formed from the decarboxylation products of ornithine and $S$-adenosylmethionine in nearly all eukaryotic cells. More recently, it has been demonstrated that arginine can be metabolised according to the same pathway leading to agmatine formation.

Cellular polyamine concentrations are highly regulated. If accumulated excessively within the cells, either due to very high extracellular amount or to deregulation of the systems, which control polyamine homeostasis, they can induce toxic effects, while polyamine depletion has been shown to inhibit cell proliferation and migration, or cause defective embryo development. These molecules are substrates of a family of enzymes that includes monoamine oxidases, diamine oxidases, polyamine oxidases, and copper containing amine oxidases. These enzymes are important because they contribute to regulate levels of polyamines. Polyamines also represent substrates for transglutaminases, enzymes which catalyze protein crosslinking by the formation of isopeptide bridges, between peptide-bound glutamines and lysines.

The physiological functions of these amines have not yet been elucidated completely at the molecular level. However, several studies have provided a better understanding of the role of these molecules in cell growth, proliferation and in pathophysiological processes. Numerous effects exercised by polyamines are well known and, in several events, the mechanisms underlying these effects have been clarified. Special attention has been given to their contribution to carcinogenesis and to the development of new approaches to the therapy of cancer and of other diseases. 
The polyamine biosynthetic pathway is very active during the growth of various cancer cells. In fact, polyamines are often present at high concentrations in rapidly dividing tumour cells and growing tissues. Among numerous other diseases, a great success in therapy has been performed in some endemic parasitic protozoa affections, as in trypanosomiasis by DFMO.

We hope that the reviews and the original papers of this issue, gathering together contributions from biochemists, pharmacologists, chemists, geneticists, molecular biologists, and clinical scientists from numerous countries, will demonstrate the current knowledge of the physiological, biochemical, and therapeutic actions of these polycationic biogenic amines, as well as providing a stimulus mainly for the new generation involved with enthusiasm and productivity in the polyamine field.

The authors and editor of the issue wish to thank professor Gert Lubec and all the people and Institutions for their kind contribution to the 11th International Congress on Amino Acids, Peptide and Proteins organization. 